Épijournal de Géométrie Algébrique

epiga.episciences.org

Volume 1 (2017), Article Nr. 8

\title{
On a theorem of Campana and Păun
}

\author{
Christian Schnell
}

\begin{abstract}
Let $X$ be a smooth projective variety over the complex numbers, and $\Delta \subseteq X$ a reduced divisor with normal crossings. We present a slightly simplified proof for the following theorem of Campana and Păun: If some tensor power of the bundle $\Omega_{X}^{1}(\log \Delta)$ contains a subsheaf with big determinant, then $(X, \Delta)$ is of log general type. This result is a key step in the recent proof of Viehweg's hyperbolicity conjecture.
\end{abstract}

Keywords. Viehweg's hyperbolicity conjecture; log general type; log cotangent bundle; foliation; movable curve class; slope semi-stability

2010 Mathematics Subject Classification. 14E99; 14F10

\section{[Français]}

\section{Titre. Sur un théorème de Campana et Păun}

Résumé. Soit $X$ une variété projective complexe lisse et $\Delta \subseteq X$ un diviseur réduit à croisements normaux. Nous présentons une démonstration légèrement simplifiée du théorème suivant de Campana et Păun : si une puissance tensorielle du fibré $\Omega_{X}^{1}(\log (\Delta))$ contient un faisceau dont le déterminant est $\operatorname{big}$, la paire $(X, \Delta)$ est alors de log-type général. Ce résultat est une étape clé dans la récente démonstration de la conjecture d'hyperbolicité de Viehweg.

Received by the Editors on April 25, 2017, and in final form on June 27, 2017.

Accepted on July 24, 2017.

Christian Schnell

Department of Mathematics, Stony Brook University, Stony Brook, NY 11794-3651, USA

e-mail: cschnell@math.stonybrook.edu

I thank Stefan Kebekus, Mihai Păun, and Mihnea Popa for their input. During the preparation of this paper, I have been supported in part by grants DMS-1404947 and DMS-1551677 from the National Science Foundation.

(C) by the author(s)

This work is licensed under http://creativecommons.org/licenses/by-sa/4.0/ 


\section{Contents}

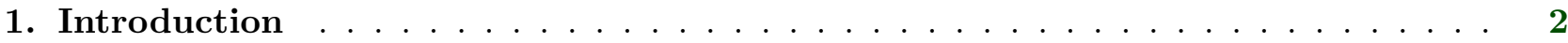

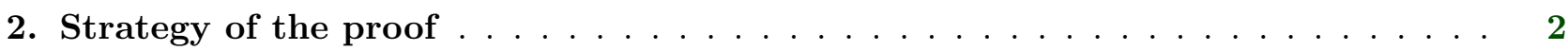

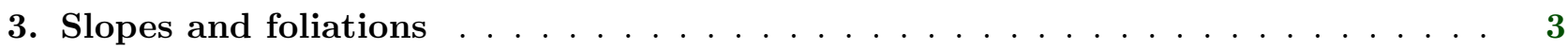

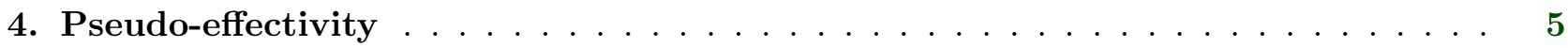

5. Induction on the dimension $\ldots \ldots \ldots \ldots \ldots$

\section{Introduction}

The purpose of this paper is to present a slightly simplified proof for the following result by Campana and Păun [CP15, Theorem 7.6]. It is a crucial step in the proof of Viehweg's hyperbolicity conjecture for families of canonically polarized manifolds [CP15, Theorem 7.13], and more generally, for smooth families of varieties of general type [PS17, Theorem A].

Theorem 1. Let $X$ be a smooth projective variety, and $\Delta \subseteq X$ a reduced divisor with at worst normal crossing singularities. If some tensor power of $\Omega_{X}^{1}(\log \Delta)$ contains a subsheaf with big determinant, then $K_{X}+\Delta$ is big.

The simplification is that I have substituted an inductive procedure for the arguments involving Campana's "orbifold cotangent bundle"; otherwise, the proof of Theorem 1 that I present here is essentially the same as in the one in [CP15]. My reason for writing this paper is that it gives me a chance to draw attention to some of the beautiful ideas involved in the proof by Campana and Păun: slope stability with respect to movable classes; a criterion for the leaves of a foliation to be algebraic subvarieties; and positivity results for relative canonical bundles.

Remark 2. The most recent arXiv version of the paper by Campana and Păun (from June 14, 2017) also contains a brief summary of our proof; see [CP15, Section 8.1].

\section{Strategy of the proof}

Let $(X, \Delta)$ be a pair, consisting of a smooth projective variety $X$ and a reduced divisor $\Delta \subseteq X$ with at worst normal crossing singularities. We denote the logarithmic cotangent bundle by the symbol $\Omega_{X}^{1}(\log \Delta)$, and its dual, the logarithmic tangent bundle, by the symbol $\mathscr{T}_{X}(-\log \Delta)$. Recall that $\mathscr{T}_{X}(-\log \Delta)$ is naturally a subsheaf of the tangent bundle $\mathscr{T}_{X}$, and that it is closed under the Lie bracket on $\mathscr{T}_{X}$. Indeed, suppose that $\Delta$ is given, in suitable local coordinates $x_{1}, x_{2}, \ldots, x_{n}$, by the equation $x_{1} x_{2} \cdots x_{k}=0$; then $\mathscr{T}_{X}(-\log \Delta)$ is generated by the $n$ commuting vector fields

$$
x_{1} \frac{\partial}{\partial x_{1}}, \ldots, x_{k} \frac{\partial}{\partial x_{k}}, \frac{\partial}{\partial x_{k+1}}, \ldots, \frac{\partial}{\partial x_{n}},
$$

and is therefore closed under the Lie bracket.

Suppose that $\Omega_{X}^{1}(\log \Delta)^{\otimes N}$ contains a subsheaf with big determinant, for some $N \geq 1$. The following observation reduces the problem to the case of line bundles.

Lemma 3. If $\Omega_{X}^{1}(\log \Delta)^{\otimes N}$ contains a subsheaf of generic rank $r \geq 1$ and with big determinant, then $\Omega_{X}^{1}(\log \Delta)^{\otimes N r}$ contains a big line bundle. 
Proof. Let $\mathscr{B} \subseteq \Omega_{X}^{1}(\log \Delta)^{\otimes N}$ be a subsheaf of generic rank $r \geq 1$, with the property that det $\mathscr{B}$ is big. After replacing $\mathscr{B}$ by its saturation, whose determinant is of course still big, we may assume that the quotient sheaf

$$
\Omega_{X}^{1}(\log \Delta)^{\otimes N} / \mathscr{B}
$$

is torsion-free, hence locally free outside a closed subvariety $Z \subseteq X$ of codimension $\geq 2$. On $X \backslash Z$, we have an inclusion of locally free sheaves

$$
\operatorname{det} \mathscr{B} \hookrightarrow \mathscr{B}^{\otimes r} \hookrightarrow \Omega_{X}^{1}(\log \Delta)^{\otimes N r},
$$

which remains valid on $X$ by Hartog's theorem.

For the purpose of proving Theorem 1, we are therefore allowed to assume that $\Omega_{X}^{1}(\log \Delta)^{\otimes N}$ contains a big line bundle $L$ as a subsheaf. Let $\mathscr{Q}$ denote the quotient sheaf, and consider the resulting short exact sequence

$$
0 \rightarrow L \rightarrow \Omega_{X}^{1}(\log \Delta)^{\otimes N} \rightarrow \mathscr{Q} \rightarrow 0 .
$$

Since $K_{X}+\Delta$ represents the first Chern class of $\Omega_{X}^{1}(\log \Delta)$, we obtain

$$
N \cdot(\operatorname{dim} X)^{N-1} \cdot\left(K_{X}+\Delta\right)=c_{1}(L)+c_{1}(\mathscr{Q})
$$

in $N^{1}(X)_{\mathbb{R}}$, the $\mathbb{R}$-linear span of codimension-one cycles modulo numerical equivalence. By assumption, the class $c_{1}(L)$ is big; Theorem 1 will therefore be proved if we manage to show that the class $c_{1}(\mathscr{Q})$ is pseudo-effective. In fact, we are going to prove the following more general result, which is of course just a special case of [CP15, Theorem 7.6 and Theorem 1.2].

Theorem 4. Let $X$ be a smooth projective variety, and $\Delta \subseteq X$ a reduced divisor with at worst normal crossing singularities. Suppose that some tensor power of $\Omega_{X}^{1}(\log \Delta)$ contains a subsheaf with big determinant. Then the first Chern class of every quotient sheaf of every tensor power of $\Omega_{X}^{1}(\log \Delta)$ is pseudo-effective.

\section{Slopes and foliations}

To simplify the presentation, we will prove Theorem 4 by contradiction. Suppose then that, for some integer $N \geq 1$, and for some quotient sheaf $\mathscr{Q}$ of $\Omega_{X}^{1}(\log \Delta)^{\otimes N}$, the class $c_{1}(\mathscr{Q})$ was not pseudoeffective. Let $\mathscr{Q}_{\text {tor }} \subseteq \mathscr{Q}$ denote the torsion subsheaf. Since

$$
c_{1}(\mathscr{Q})=c_{1}\left(\mathscr{Q}_{\text {tor }}\right)+c_{1}\left(\mathscr{Q} / \mathscr{Q}_{\text {tor }}\right)
$$

and since $c_{1}\left(\mathscr{Q}_{\text {tor }}\right)$ is effective, we may replace $\mathscr{Q}$ by $\mathscr{Q} / \mathscr{Q}_{\text {tor }}$, and assume without any loss of generality that $\mathscr{Q}$ is torsion-free (and nonzero).

By the characterization of the pseudo-effective cone in [BDPP13, Theorem 2.2], there is a movable class $\alpha \in N_{1}(X)_{\mathbb{R}}$ such that $c_{1}(\mathscr{Q}) \cdot \alpha<0$. As shown in [CP11, GKP16], there is a good theory of $\alpha$-semistability for torsion-free sheaves, with almost all the properties that are familiar from the case of complete intersection curves. We use this theory freely in what follows. By assumption,

$$
\mu_{\alpha}(\mathscr{Q})=\frac{c_{1}(\mathscr{Q}) \cdot \alpha}{\operatorname{rk} \mathscr{Q}}<0,
$$

and so $\mathscr{Q}$ is a torsion-free quotient sheaf of $\Omega_{X}^{1}(\log \Delta)^{\otimes N}$ with negative $\alpha$-slope. The dual sheaf $\mathscr{Q}^{*}$ is therefore a saturated subsheaf of $\mathscr{T}_{X}(-\log \Delta)^{\otimes N}$ with positive $\alpha$-slope. At this point, we recall the following result about tensor products. 
Theorem 5. Let $\alpha \in N_{1}(X)_{\mathbb{R}}$ be a movable class. If $\mathscr{F}$ and $\mathscr{G}$ are torsion-free and $\alpha$-semistable coherent sheaves on $X$, then their tensor product

$$
\mathscr{F} \hat{\otimes} \mathscr{G}=(\mathscr{F} \otimes \mathscr{G}) /(\mathscr{F} \otimes \mathscr{G})_{\text {tor }},
$$

modulo torsion, is again $\alpha$-semistable, and $\mu_{\alpha}(\mathscr{F} \hat{\otimes} \mathscr{G})=\mu_{\alpha}(\mathscr{F})+\mu_{\alpha}(\mathscr{G})$.

Proof. For the reflexive hull of the tensor product, this is proved in [GKP16, Theorem 4.2 and Proposition 4.4], based on analytic results by Toma [CP11, Appendix]. Since $\mathscr{F} \hat{\otimes} \mathscr{G}$ and its reflexive hull are isomorphic outside a closed subvariety of codimension $\geq 2$, the assertion follows. (The formula for the $\alpha$-slope of $\mathscr{F} \hat{\otimes} \mathscr{G}$ is of course valid for arbitrary nonzero torsion-free coherent sheaves $\mathscr{F}$ and G.)

Similarly, the fact that $\mathscr{T}_{X}(-\log \Delta)^{\otimes N}$ has a subsheaf with positive $\alpha$-slope implies, again by [GKP16, Theorem 4.2 and Proposition 4.4], that $\mathscr{T}_{X}(-\log \Delta)$ must also contain a subsheaf with positive $\alpha$-slope. Let $\mathscr{F}_{\Delta} \subseteq \mathscr{T}_{X}(-\log \Delta)$ be the maximal $\alpha$-destabilizing subsheaf [GKP16, Corollary 2.24].

Lemma 6. $\mathscr{F}_{\Delta}$ is a saturated, $\alpha$-semistable subsheaf of $\mathscr{T}_{X}(-\log \Delta)$, of positive $\alpha$-slope. Every subsheaf of $\mathscr{T}_{X}(-\log \Delta) / \mathscr{F}_{\Delta}$ has $\alpha$-slope less than $\mu_{\alpha}\left(\mathscr{F}_{\Delta}\right)$.

Proof. This is clear from the construction of the maximal destabilizing subsheaf in [GKP16, Corollary 2.4]. Note that $\mathscr{F}_{\Delta}$ is the first step in the Harder-Narasimhan filtration of $\mathscr{T}_{X}(-\log \Delta)$, see [GKP16, Corollary 2.26].

Recall that we have an inclusion $\mathscr{T}_{X}(-\log \Delta) \subseteq \mathscr{T}_{X}$. We define another coherent subsheaf $\mathscr{F} \subseteq$ $\mathscr{T}_{X}$ as the saturation of $\mathscr{F}_{\Delta}$ in $\mathscr{T}_{X}$; then $\mathscr{T}_{X} / \mathscr{F}$ is torsion-free, and

$$
\mathscr{F} \cap \mathscr{T}_{X}(-\log \Delta)=\mathscr{F}_{\Delta} .
$$

We will see in a moment that $\mathscr{F}$ is actually a (typically, singular) foliation on $X$. Recall that, in general, a foliation on a smooth projective variety is a saturated subsheaf $\mathscr{F} \subseteq \mathscr{T}_{X}$ that is closed under the Lie bracket on $\mathscr{T}_{X}$. From the Lie bracket, one constructs an $\mathscr{O}_{X}$-linear mapping

$$
N: \mathscr{F} \hat{\otimes} \mathscr{F} \rightarrow \mathscr{T}_{X} / \mathscr{F},
$$

called the O'Neil tensor of $\mathscr{F}$; evidently, $\mathscr{F}$ is a foliation if and only if its O'Neil tensor vanishes.

Lemma 7. The O'Neil tensor

$$
N: \mathscr{F} \hat{\otimes} \mathscr{F} \rightarrow \mathscr{T}_{X} / \mathscr{F}
$$

vanishes, and $\mathscr{F}$ is therefore a foliation on $X$.

Proof. The Lie bracket of two sections of $\mathscr{T}_{X}(-\log \Delta)$ is a section of $\mathscr{T}_{X}(-\log \Delta)$, and so we get a logarithmic O'Neil tensor

$$
N_{\Delta}: \mathscr{F}_{\Delta} \hat{\otimes} \mathscr{F}_{\Delta} \rightarrow \mathscr{T}_{X}(-\log \Delta) / \mathscr{F}_{\Delta}
$$

The key point is that $N_{\Delta}=0$. Indeed, by Theorem 5 , the tensor product $\mathscr{F}_{\Delta} \hat{\otimes} \mathscr{F}_{\Delta}$, modulo torsion, is again $\alpha$-semistable of slope

$$
\mu_{\alpha}\left(\mathscr{F}_{\Delta} \hat{\otimes} \mathscr{F}_{\Delta}\right)=2 \cdot \mu_{\alpha}\left(\mathscr{F}_{\Delta}\right)>\mu_{\alpha}\left(\mathscr{F}_{\Delta}\right),
$$

which is strictly greater than the slope of any nonzero subsheaf of $\mathscr{T}_{X}(-\log \Delta) / \mathscr{F}_{\Delta}$ by Lemma 6 . This inequality among slopes implies that $N_{\Delta}=0$, see for instance [GKP16, Proposition 2.16 and Corollary 2.17]. 
The O'Neil tensor $N$ and the logarithmic O'Neil tensor $N_{\Delta}$ are both induced by the Lie bracket on $\mathscr{T}_{X}$, and so we have the following commutative diagram:

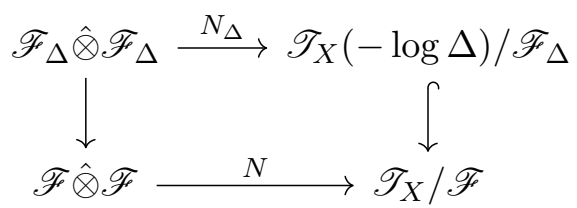

The vertical arrow on the right is injective by (3.2). Now $N_{\Delta}=0$ implies that $N$ factors through the cokernel of the vertical arrow on the left; but the cokernel is a torsion sheaf, whereas $\mathscr{T}_{X} / \mathscr{F}$ is torsion-free. The conclusion is that $N=0$.

The next step in the proof is to show that the foliation $\mathscr{F}$ is actually algebraic. This is a simple consequence of the powerful algebraicity theorem of Campana and Păun [CP15, Theorem 1.1], which generalizes a well-known result by Bogomolov and McQuillan [BM16] and Bost [Bos01, §3.3] from complete intersection curves to movable classes. (See also the paper [KST07] by Kebekus, Solà Conde, and Toma.)

Theorem 8. Let $X$ be a smooth projective variety over the complex numbers, and let $\mathscr{F} \subseteq \mathscr{T}_{X}$ be a foliation. Suppose that there exists a movable class $\alpha \in N_{1}(X)_{\mathbb{R}}$, such that every nonzero quotient sheaf of $\mathscr{F}$ has positive $\alpha$-slope. Then $\mathscr{F}$ is an algebraic foliation, and its leaves are rationally connected.

To apply this in our setting, we observe that every quotient sheaf of $\mathscr{F}$ is, at least over the open subset $X \backslash \Delta$, also a quotient sheaf of $\mathscr{F}_{\Delta}$, because $\mathscr{F}$ and $\mathscr{F}_{\Delta}$ agree outside the divisor $\Delta$. As $\mathscr{F}_{\Delta}$ is $\alpha$-semistable with $\mu_{\alpha}(\mathscr{F})>0$, it follows easily that every quotient sheaf of $\mathscr{F}$ has positive $\alpha$-slope. We can now invoke Theorem 8 and conclude that the foliation $\mathscr{F}$ is algebraic. In other words [CP15, $\S 4]$, there exists a dominant rational mapping

$$
p: X \rightarrow Z
$$

to a smooth projective variety $Z$, such that

$$
\mathscr{F}=\operatorname{ker}\left(d p: \mathscr{T}_{X} \rightarrow p^{*} \mathscr{T}_{Z}\right)
$$

outside a subset of codimension $\geq 2$. More precisely, let us follow [CKT16, Construction 2.29] and denote by the symbol $\mathscr{T}_{X / Z}$ the unique reflexive sheaf on $X$ that agrees with $\operatorname{ker}\left(d p: \mathscr{T}_{X} \rightarrow p^{*} \mathscr{T}_{Z}\right)$ on the big open subset where $p$ is a morphism. Using this notation, the algebraicity of $\mathscr{F}$ may be expressed as

$$
\mathscr{F}=\mathscr{T}_{X / Z}
$$

indeed, $\mathscr{F}$ is reflexive, due to the fact that $\mathscr{T}_{X} / \mathscr{F}$ is torsion-free.

Remark 9. Theorem 8 also says that the fibers of $p$ are rationally connected, but we are not going to make any use of this extra information. This means that the proof of Theorem 4 only uses characteristic zero methods.

\section{Pseudo-effectivity}

Let us first convince ourselves that $Z$ cannot be a point. This will later allow us to argue by induction on the dimension, because the general fiber of $p$ has dimension less than $\operatorname{dim} X$.

Lemma 10. With notation as above, we must have $\operatorname{dim} Z \geq 1$. 
Proof. If $\operatorname{dim} Z=0$, then $\mathscr{F}=\mathscr{T}_{X}$ and $\mathscr{F}_{\Delta}=\mathscr{T}_{X}(-\log \Delta)$, and consequently, the logarithmic tangent bundle $\mathscr{T}_{X}(-\log \Delta)$ is $\alpha$-semistable of positive slope. Since the tensor product of $\alpha$ semistable sheaves remains $\alpha$-semistable [GKP16, Proposition 4.4], this means that any tensor power of $\Omega_{X}^{1}(\log \Delta)$ is $\alpha$-semistable of negative slope. But that contradicts the hypothesis of Theorem 4, namely that some tensor power of $\Omega_{X}^{1}(\log \Delta)$ contains a subsheaf with big determinant, because the $\alpha$-slope of such a subsheaf is obviously positive.

The only properties of $\mathscr{F}_{\Delta}$ that we are still going to use in the proof of Theorem 4 are the identity in (3.2), and the fact that $c_{1}\left(\mathscr{F}_{\Delta}\right) \cdot \alpha>0$ for a movable class $\alpha \in N_{1}(X)_{\mathbb{R}}$. In return, we are allowed to assume that $p: X \rightarrow Z$ is a morphism.

Lemma 11. Without loss of generality, $p: X \rightarrow Z$ is a morphism.

Proof. Choose a birational morphism $f: \tilde{X} \rightarrow X$, for example by resolving the singularities of the closure of the graph of $p: X \rightarrow Z$ inside $X \times Z$, with the following properties: the rational mapping $p \circ f$ extends to a morphism $\tilde{p}: \tilde{X} \rightarrow Z$; both $K_{\tilde{X} / X}$ and $\tilde{p}^{*} \Delta$ are normal crossing divisors; and $f$ is an isomorphism over the open subset where $p$ is already a morphism.

Let $\tilde{\Delta}$ be the reduced normal crossing divisor whose support is equal to the preimage of $\Delta$ in $\tilde{X}$. Then

$$
\Omega_{\tilde{X}}^{1}(\log \tilde{\Delta}) \cong \tilde{p}^{*} \Omega_{X}^{1}(\log \Delta),
$$

and since the pullback of a big line bundle by $\tilde{p}$ stays big, it is still true that some tensor power of $\Omega_{\tilde{X}}^{1}(\log \tilde{\Delta})$ contains a big line bundle as a subsheaf. Now define

$$
\tilde{\mathscr{F}}=\mathscr{T}_{\tilde{X} / Z}=\operatorname{ker}\left(\tilde{p}^{*}: \mathscr{T}_{\tilde{X}} \rightarrow \tilde{p}^{*} \mathscr{T}_{Z}\right)
$$

which is a saturated subsheaf of $\mathscr{T}_{\tilde{X}}$. The intersection

$$
\tilde{\mathscr{F}} \cap \mathscr{T}_{\tilde{X}}(-\log \tilde{\Delta})
$$

is a saturated (and hence reflexive) subsheaf of $\mathscr{T}_{\tilde{X}}(-\log \tilde{\Delta})$, whose pushforward to $X$ is isomorphic to $\mathscr{F}_{\Delta}$, by $(3.2)$ and the fact that $\mathscr{F}_{\Delta}$ is reflexive. Consequently,

$$
c_{1}\left(\tilde{\mathscr{F}} \cap \mathscr{T}_{\tilde{X}}(-\log \tilde{\Delta})\right) \cdot \tilde{\alpha}=c_{1}\left(\mathscr{F}_{\Delta}\right) \cdot \alpha>0,
$$

where the class $\tilde{\alpha}=\tilde{p}^{*} \alpha \in N_{1}(\tilde{X})_{\mathbb{R}}$ is of course still movable. Nothing essential is therefore changed if we replace the rational mapping $p: X \rightarrow Z$ by the morphism $\tilde{p}: \tilde{X} \rightarrow Z$; the divisor $\Delta \subseteq X$ by $\tilde{\Delta} \subseteq \tilde{X}$; the sheaf $\mathscr{F}_{\Delta}$ by the intersection

$$
\mathscr{T}_{\tilde{X} / Z} \cap \mathscr{T}_{\tilde{X}}(-\log \tilde{\Delta}) \subseteq \mathscr{T}_{\tilde{X}}
$$

and the movable class $\alpha \in N_{1}(X)_{\mathbb{R}}$ by its pullback $\tilde{\alpha}=\tilde{p}^{*} \alpha$.

Let $R(p)$ denote the ramification divisor of the morphism $p: X \rightarrow Z$; see [CKT16, Definition 2.16] for the precise definition. Recall from [CKT16, Lemma 2.31] the following formula for the first Chern class of our foliation $\mathscr{F} \subseteq \mathscr{T}_{X}$, in $N^{1}(X)_{\mathbb{R}}$ :

$$
c_{1}(\mathscr{F})=c_{1}\left(\mathscr{T}_{X / Z}\right)=-K_{X / Z}+R(p)
$$

Computing the first Chern class of $\mathscr{F}_{\Delta}$ is a little tricky [CP15, Proposition 5.1], but at least we can use the fact that $\mathscr{F}=\mathscr{T}_{X / Z}$ to estimate the difference

$$
c_{1}(\mathscr{F})-c_{1}\left(\mathscr{F}_{\Delta}\right)=c_{1}\left(\mathscr{F} / \mathscr{F}_{\Delta}\right) .
$$

Recall that the horizontal part $\Delta^{\text {hor }} \subseteq \Delta$ is the union of all irreducible components of $\Delta$ that map onto $Z$; evidently, $\Delta^{\text {hor }}$ is again a reduced divisor on $X$ with at worst normal crossing singularities. 
Lemma 12. The class $c_{1}(\mathscr{F})-c_{1}\left(\mathscr{F}_{\Delta}\right)-\Delta^{\text {hor }}$ is effective.

Proof. It is easy to see from (3.2) that we have an inclusion of sheaves

$$
\mathscr{F} / \mathscr{F}_{\Delta} \hookrightarrow \mathscr{T}_{X} / \mathscr{T}_{X}(-\log \Delta) .
$$

The sheaf on the right-hand side is supported on the divisor $\Delta$, and a brief computation shows that

$$
\mathscr{T}_{X} / \mathscr{T}_{X}(-\log \Delta) \cong \bigoplus_{D \subseteq \Delta} \mathscr{N}_{D \mid X}
$$

is isomorphic to the direct sum of the normal bundles of the irreducible components of $\Delta$. The rank of $\mathscr{F} / \mathscr{F}_{\Delta}$ at the generic point of $D$ is thus either 0 or 1 , and

$$
c_{1}\left(\mathscr{F} / \mathscr{F}_{\Delta}\right)=\sum_{D \subseteq \Delta} a_{D} D
$$

where $a_{D}=0$ if $\mathscr{F}=\mathscr{F} \Delta$ at the generic point of $D$, and $a_{D}=1$ otherwise. To prove that $c_{1}\left(\mathscr{F} / \mathscr{F}_{\Delta}\right)-$ $\Delta^{\text {hor }}$ is effective, we only have to argue that $\mathscr{F} \neq \mathscr{F} \Delta$ at the generic point of each irreducible component of $\Delta^{\text {hor }}$. This is a consequence of the fact that $\mathscr{F}=\mathscr{T}_{X / Z}$, as we now explain.

Fix an irreducible component $D$ of the horizontal part $\Delta^{\text {hor }}$. At the generic point of $D$, the morphism $p: X \rightarrow Z$ is smooth. After choosing suitable local coordinates $x_{1}, \ldots, x_{n}$ in a neighborhood of a sufficiently general point of $D$, we may therefore assume that $p$ is locally given by

$$
p\left(x_{1}, \ldots, x_{n}\right)=\left(x_{1}, \ldots, x_{d}\right),
$$

where $d=\operatorname{dim} Z$, and that the divisor $\Delta$ is defined by the equation $x_{n}=0$. In these local coordinates, $\mathscr{F}=\mathscr{T}_{X / Z}$ is the subbundle of $\mathscr{T}_{X}$ spanned by

$$
\frac{\partial}{\partial x_{n}}, \frac{\partial}{\partial x_{n-1}}, \ldots, \frac{\partial}{\partial x_{d+1}} .
$$

On the other hand, the subsheaf $\mathscr{T}_{X}(-\log \Delta)$ is spanned by the vector fields

$$
x_{n} \frac{\partial}{\partial x_{n}}, \frac{\partial}{\partial x_{n-1}}, \ldots, \frac{\partial}{\partial x_{d+1}}, \ldots, \frac{\partial}{\partial x_{1}},
$$

and so it is clear from (3.2) that $\mathscr{F} \neq \mathscr{F}_{\Delta}$ in a neighborhood of the given point.

From Lemma 12, we draw the conclusion that

$$
-\left(K_{X / Z}+\Delta^{h o r}-R(p)\right) \cdot \alpha=\left(c_{1}(\mathscr{F})-\Delta^{h o r}\right) \cdot \alpha \geq c_{1}\left(\mathscr{F}_{\Delta}\right) \cdot \alpha>0,
$$

where $\alpha \in N_{1}(X)_{\mathbb{R}}$ is the movable class from above. We will therefore reach the desired contradiction if we manage to prove that the divisor class $K_{X / Z}+\Delta^{\text {hor }}-R(p)$ is pseudo-effective. According to [CP15, Theorem 3.3] or to [CKT16, Theorem 7.1], it is actually enough to check that $K_{F}+\Delta_{F}$ is pseudo-effective for a general fiber $F$ of the morphism $p$; and we can prove, by induction on the dimension, that $K_{F}+\Delta_{F}$ is not only pseudo-effective, but even big. The results that we use here are slight improvements of [Cam04, Theorem 4.13], which is itself a generalization of Viehweg's weak positivity theorem. 


\section{Induction on the dimension}

In this section, we use induction on the dimension to finish the proof of Theorem 4 and Theorem 1.

Proposition 13. Suppose that Theorem 1 is true in dimension less than $\operatorname{dim} X$. If some tensor power of $\Omega_{X}^{1}(\log \Delta)$ contains a subsheaf with big determinant, then $K_{X / Z}+\Delta^{\text {hor }}$ is pseudo-effective.

Proof. Let $F$ be a general fiber of the morphism $p: X \rightarrow Z$; $\operatorname{since} \operatorname{dim} Z \geq 1$, we have $\operatorname{dim} F \leq$ $\operatorname{dim} X-1$. Denote by $\Delta_{F}$ the restriction of $\Delta$; since $F$ is a general fiber, $\Delta_{F}$ is still a normal crossing divisor. Clearly

$$
\left.\left(K_{X / Z}+\Delta^{h o r}\right)\right|_{F}=K_{F}+\Delta_{F}
$$

and according to [CKT16, Theorem 7.3], the pseudo-effectivity of $K_{X / Z}+\Delta^{\text {hor }}$ will follow if we manage to show that $K_{F}+\Delta_{F}$ is pseudo-effective.

By hypothesis and by Lemma 3, there is a nonzero morphism

$$
L \rightarrow \Omega_{X}^{1}(\log \Delta)^{\otimes k}
$$

from a big line bundle $L$ to some tensor power of $\Omega_{X}^{1}(\log \Delta)$. Since $F$ is a general fiber of $p: X \rightarrow Z$, we can restrict this morphism to $F$ to obtain a nonzero morphism

$$
L_{F} \rightarrow\left(\left.\Omega_{X}^{1}(\log \Delta)\right|_{F}\right)^{\otimes k}
$$

Here $L_{F}$ denotes the restriction of $L$ to the fiber; since $L$ is big, $L_{F}$ is also big.

The inclusion of $F$ into $X$ gives rise to a short exact sequence

$$
\left.0 \rightarrow \mathscr{N}_{F \mid X} \rightarrow \Omega_{X}^{1}(\log \Delta)\right|_{F} \rightarrow \Omega_{F}^{1}\left(\log \Delta_{F}\right) \rightarrow 0,
$$

which induces a filtration on the $k$-th tensor power of the locally free sheaf in the middle. Since the normal bundle $\mathscr{N}_{F \mid X}$ is trivial of $\operatorname{rank} \operatorname{dim} Z$, we find, by looking at the subquotients of this filtration, that there is a nonzero morphism

$$
L_{F} \rightarrow \Omega_{F}^{1}\left(\log \Delta_{F}\right)^{\otimes j}
$$

for some $0 \leq j \leq k$. Because $L_{F}$ is big, we actually have $1 \leq j \leq k$. Since we are assuming that Theorem 1 is true for the pair $\left(F, \Delta_{F}\right)$, the class $K_{F}+\Delta_{F}$ is big on $F$, hence pseudo-effective. Appealing to [CKT16, Theorem 7.3], we deduce that the class $K_{X / Z}+\Delta^{\text {hor }}$ is pseudo-effective on $X$.

By induction on the dimension, the two assumptions of Proposition 13 are met in our case, and the class $K_{X / Z}+\Delta^{h o r}$ is therefore pseudo-effective. According to [CKT16, Theorem 7.1], this implies that $K_{X / Z}+\Delta^{\text {hor }}-R(p)$ is also pseudo-effective. ${ }^{1}$ Going back to the inequality in (4.5), we find that

$$
0 \geq-\left(K_{X / Z}+\Delta^{h o r}-R(p)\right) \cdot \alpha \geq c_{1}\left(\mathscr{F}_{\Delta}\right) \cdot \alpha>0,
$$

and so we have reached the desired contradiction. The conclusion is that $c_{1}(\mathscr{Q})$ is indeed pseudoeffective, and so Theorem 4 and Theorem 1 are proved.

Remark 14. Most of the argument, for example the proof of Lemma 10, goes through when some tensor power of $\Omega_{X}^{1}(\log \Delta)$ contains a subsheaf with pseudo-effective determinant. But Theorem 4 is obviously not true under this weaker hypothesis: for example, on the product $E \times \mathbb{P}^{1}$ of an elliptic curve and $\mathbb{P}^{1}$, there are nontrivial one-forms, yet the canonical bundle is not pseudo-effective. What happens is that the last step in the proof of Proposition 13 breaks down: when $L$ is not big, it may be that $j=0$ (and $L_{F}$ is then trivial).

$1 \uparrow$ As stated, both [CP15, Theorem 3.3] and [CKT16, Theorem 7.1] actually assume that $K_{X}+\Delta$ is pseudoeffective, but in the case of a morphism $p: X \rightarrow Z$, the proofs go through under the weaker hypothesis that $K_{X / Z}+\Delta^{\text {hor }}$ is pseudo-effective. 


\section{References}

[BDPP13] Sébastien Boucksom, Jean-Pierre Demailly, Mihai Păun, and Thomas Peternell, The pseudo-effective cone of a compact Kähler manifold and varieties of negative Kodaira dimension, J. Algebraic Geom. 22 (2013), no. 2, 201-248. MR-3019449

[BM16] Fedor Bogomolov and Michael McQuillan, Rational curves on foliated varieties. In: Foliation Theory in Algebraic Geometry (Paolo Cascini, James McKernan, and Jorge Vitório Pereira, eds.), pp. 21-51, Springer International Publishing, Cham, 2016. ihes/M01-07

[Bos01] Jean-Benoît Bost, Algebraic leaves of algebraic foliations over number fields, Publ. Math. Inst. Hautes Études Sci. (2001), no. 93, 161-221. MR-1863738

[Cam04] Frédéric Campana, Orbifolds, special varieties and classification theory, Ann. Inst. Fourier (Grenoble) 54 (2004), no. 3, 499-630. MR-2097416

[CKT16] Benoît Claudon, Stefan Kebekus, and Behrouz Taji, Generic positivity and applications to hyperbolicity of moduli spaces, preprint 2016. arXiv:1610.09832

[CP11] Frédéric Campana and Thomas Peternell, Geometric stability of the cotangent bundle and the universal cover of a projective manifold, with an appendix by Matei Toma, Bull. Soc. Math. France 139 (2011), no. 1, 41-74. MR-2815027

[CP15] Frédéric Campana and Mihai Păun, Foliations with positive slopes and birational stability of orbifold cotangent bundles, preprint 2015. arXiv:1508.02456

[GKP16] Daniel Greb, Stefan Kebekus, and Thomas Peternell, Movable curves and semistable sheaves, Int. Math. Res. Not. IMRN (2016), no. 2, 536-570. MR-3493425

[KST07] Stefan Kebekus, Luis Solá Conde, and Matei Toma, Rationally connected foliations after Bogomolov and McQuillan, J. Algebraic Geom. 16 (2007), no. 1, 65-81. MR-2257320

[PS17] Mihnea Popa and Christian Schnell, Viehweg's hyperbolicity conjecture for families with maximal variation, Invent. Math. 208 (2017), no. 3, 677-713. MR-3648973 